\title{
Ingeniería kanseiaplicada a un estudio referido a cinco maderas comerciales de Chile
}

\section{Kansei engineering applied to studies referred to commercial woods in Chile}

\author{
Jimena Alarcón Castrol*, Andrea Llorens Vargas’ y Gino Ormeño Bustos'
}

\footnotetext{
Universidad del Bío-Bío, Chile. Grupo de Investigación en Diseño. Concepción, Chile

* Autora de correspondencia. jimenaal@gmail.com
}

\section{RESUMEN}

Esta investigación tuvo como propósito conocer la percepción emocional que los proyectistas tienen en relación con la madera de las cinco especies comerciales más vendidas de Chile, para establecer conclusiones referidas a su calidad percibida. El estudio busca definir valoraciones comparativas y jerárquicas preferenciales entre las especies y conocer las cualidades superficiales relevantes de la madera que influyen en la satisfacción usuaria. Regularmente, los criterios de elegibilidad para aplicaciones estéticas se centran en el proyectista, correspondiéndole tomar decisiones para idear configuraciones espaciales destinadas a satisfacer a los usuarios finales. A través de este estudio, el proyectista adquiere nueva información útil para valorar las emociones que el espacio habitable podría provocar en sus futuros ocupantes, basado en la utilización de la madera de estas especies. El fundamento metodológico se centra en la aplicación de la ingeniería kansei, disciplina encargada de establecer las relaciones entre las emociones que un determinado estímulo genera en los seres humanos, en este caso, la presencia de la madera. Se realizó un estudio a una muestra no probabilística intencionada de expertos del área proyectual, mediante uso de cuestionarios con opciones cuantificables y preguntas estructuradas acorde con ejes semánticos. Como resultado se obtuvieron respuestas relativas a las valoraciones de las maderas y se generó un método de recogida de datos extrapolable a estudios relativos a otros tipos de maderas comerciales. La conclusión específica consiste en que las maderas oscuras y sin nudos son mejor valoradas emocionalmente por los usuarios.

PALABRAS CLAVE: calidad percibida, cualidades superficiales de la madera, emociones, hábitat, madera, satisfacción del usuario.

\begin{abstract}
This research had the objective to know the emotional perception of designers about the five most sold commercial woods in Chile, to establish conclusions referred to perceived quality. The study seeks to assess comparative and hierarchical preferential valuations among species and learn about relevant visual characteristics of wood with influence in user satisfaction. Usually, the eligibility criteria for aesthetic applications is focused on the designer, assigning him/her the task of making decisions to devise spatial configurations designed to meet end users requirements, who are making use of these facilities. Through this study, the designer acquires new useful information to assess emotions the living space could cause in future occupants, based on the use of wood species. The methodological foundation focuses on kansei engineering application, a discipline responsible of establishing relationships between the emotions that a given stimulus generates in human beings, in this case, the presence of wood. The study was carried out on an intentional non-probabilistic sample of experts from the design area, using survey with quantifiable options and structured questions according to semantic axes. As result, the answers obtained were referred to wood valuations and a data collection method that can be extrapolated to studies related to other types of commercial wood was generated. The specific conclusion is that dark, knotless woods are better valued emotionally by users.
\end{abstract}

KEYWORDS: perceived quality, superficial qualities of the wood, emotions, habitat, wood, user satisfaction. 


\section{INTRODUCCIÓN}

La investigación se centra en el estudio perceptual y emocional de la madera de cinco especies comerciales de Chile, aplicando parámetros metodológicos provenientes del ámbito de la ingeniería kansei, término japonés que significa kan: sensación, sensibilidad, sentir, emoción y sei: sensitivo, sentidos, impresión, apreciación (Nagamachi, 2011); siendo la disciplina encargada de establecer las relaciones entre las emociones que un determinado producto genera en los seres humanos (Nagamachi, 1995, Centro Tecnológico para el Diseño y la Producción Industrial de Asturias [Prodintec], 2011). En este marco, el término kansei es utilizado para hacer referencia al modo como las representaciones de objetos y sus funcionalidades, son convertidas en imágenes emocionales en un espacio de organización mental interior. Fiore y Kimle (1997) mencionan que la visión tiende a dominar la experiencia humana y que, al establecer un estudio comparativo de la importancia de las modalidades sensoriales, las personas evalúan que la vista es muy importante para la valoración que se establece respecto de los estímulos (Schifferstein, 2006). En este sentido, los productos orientados a conformar parte relevante del espacio habitable y que consideran cambios en la percepción de los mismos, deben ser evaluados para generar una interacción que facilite una experiencia multisensorial con una cierta calidad (Özcan y van Egmond, 2009; Schifferstein y Hekkert, 2011; Bedolla, 2003).

El rol de las emociones en las interacciones entre humanos y productos, así como los beneficios del diseño que evoca emociones positivas se han discutido ampliamente en la literatura relativa a diseño (Rognoli y Levi, 2005; Rognoli, 2010; Desmet y Sääksjärvi, 2016). Comprensiones sobre las emociones del usuario han demostrado ser relevantes para el diseño debido a que influyen fuertemente en el comportamiento de uso (Tractinsky, Katz y Ikar, 2000) y la riqueza de las experiencias (Fokkinga y Desmet, 2012). Para Schuller y Parks (2014), una de las cinco categorías para probar que las emociones influyen en la satisfacción de los individuos, es a través de vivenciar experiencias y sensaciones, por lo que es relevante conocer el significado de éstas en las personas (Valencia, Mugge, Schoormans y Schifferstein, 2014). Para la presente investigación, la ingeniería afectiva proporciona la metodología para medir las respuestas afectivas de los proyectistas respecto a las cualidades superficiales de la madera, las que generan diversas emociones en los individuos (Osgood, Suci y Tannenbaum, 1957).

Las cualidades superficiales de la madera son diferentes dependiendo de su especie, influyendo en ello las condiciones de crecimiento diferenciado del árbol y las propiedades biológicas compuestas principalmente por moléculas de celulosa y lignina. La clasificación por aspecto (Instituto Nacional de Normalización, 1974; Viña, 1997; Chávez, Hernández y Ruiz, 2010), que básicamente define estas cualidades, agrupa estas singularidades considerando la etiología del defecto, ya sea de tipo estructural o de procedimiento. Entre ellas se encuentran: 1. Textura visual, entendida como el atributo de una superficie estrictamente bidimensional (Wong, 1991), caracterizada por el tamaño de los elementos físicos externos (vasos en el caso de latifoliadas y traqueidas cuando se trata de coníferas), que varían de grueso a fino o de tosco a homogéneo; 2. Patrón decorativo, definido por la dirección de la fibra con respecto al eje del tronco, que pueden ser rectos e inclinados, representado por el dibujo resultante del corte en la superficie de la madera, pudiendo ser liso, que proviene de una madera con textura fina y homogénea; rayado propio de la madera con corte tangencial o floreado (o en tablas tangenciales de arcos superpuestos), producto de la presencia de textura heterogénea, donde los anillos de crecimiento se visualizan claramente; siendo el jaspeado (punteado en tablas tangenciales), producto de la presencia de radios leñosos conspicuos en la madera; mientras que el espigado es por presencia de grano entrelazado, angular con respecto al eje del árbol.

Las características otorgadas por vetas, son dependientes del curso de los anillos de crecimiento recorrido de las fibras y radios medulares; 3. Color superficial, determinado por las sustancias químicas integrantes de las células, las proporciones de lignina, 
celulosa y el grado de mineralización. Se consideró dentro de este ítem, por una parte, la influencia de manchas generadas por hongos, concentraciones de resina o cualquier agente que en general influyera sobre el color superficial; y, de manera especial, se realizó una evaluación referida a las tonalidades oscura y clara, ya que la investigación hace énfasis en este aspecto como discriminador de especies por parte de los usuarios. La mayoría de las maderas duras presentan color oscuro si se refiere al duramen, ya que su albura normalmente es más clara; mientras que las maderas blandas, esencialmente de coníferas, poseen en su mayoría un color más bien pálido.; 4. Brillo, es natural y su intensidad varía según la especie, dirección del corte de la pieza de árbol, presencia de extractivos en la madera, así como también, de las características anatómicas de las células que la componen; por último, 5. Presencia de nudos, que es una porción de madera dura y compacta perteneciente a las ramas que quedaron incluidas en el tronco, se encuentran comúnmente como elemento que dificulta el uso de la madera y pone a prueba la aceptación de los usuarios como elemento decorativo incrustado en ella.

Todos los aspectos que definen las cualidades superficiales de la madera, influyen en la emoción y calidad percibida por los usuarios. En este sentido, el valor visual del material, interviene en la percepción del espacio en que comparece. Según Goleman (2003), en oportunidades se diseña sin tomar en consideración las emociones, las características de personalidad, el tipo de cultura de origen de quienes posteriormente ocuparán las dependencias proyectadas, lo que podría conducir a un error, debido al desencuentro entre lo ideado y la preferencia de quienes harán uso de esa construcción. Numerosos bienes de consumo son susceptibles de ser percibidos desde la totalidad de los sentidos, de modo que las emociones que transmiten a los consumidores afectan inevitablemente su decisión final de adquirirlo o rechazarlo (Villanueva, Sampedro, de Ávila y González, 2013). En este sentido, la concepción de entornos capaces de transmitir una información predefinida o lograr un efecto específico en los consumidores, puede tener un gran impacto económico y a nivel de satisfactores (Jordan, 2000). Según Stein y Meredith (1993), la experiencia perceptual del mundo ocurre de diversos modos: sensoriales, afectivos y simbólicos, mientras que investigaciones prueban que algunas caracterizaciones superficiales inherentes a objetos de consumo de distintas funcionalidades, son percibidas bajo iguales o similares valoraciones por los usuarios. Por ejemplo, el color y el material contribuyen igualmente a la experiencia 'de calor' en bufandas y bandejas (Fenko, Schifferstein y Hekkert, 2008), mientras que la experiencia 'de frescura' en los líquidos limpiadores, depende más del olor que del color (Fenko, Schifferstein y Hekkert,, 2011); y los sonidos de electrodomésticos influyen positiva o negativamente en la idea de calidad que transfieren (Ludden y Schifferstein, 2007). Estos resultados sugieren que, para crear una experiencia agradable, los proyectistas tengan que prestar atención a las propiedades sensoriales de los materiales (Desmet y Hekkert, 2007; Desmet y Hekkert, 2009). En esta medida, todos los sentidos pueden ser estimulados en diversos grados y, a través de ello, valorarse los significados y emociones que han surgido debido a ese contacto con el material o entorno en cuestión (Hekkert, Snelders y van Wieringer, 2003). Entonces, parece interesante definir con certeza asuntos relativos a la percepción de la madera como material, de manera que los proyectistas puedan considerar esta información al momento de tomar decisiones relativas a su presencia visual en entornos habitables.

\section{OBJETIVOS}

La investigación considera dentro de sus objetivos, definir los factores emocionales vinculados a las cualidades superficiales de la madera, que influyen en los satisfactores perceptuales de los proyectistas. En este caso, el foco se centra en el estudio de la madera de cinco especies comerciales de Chile, empleando métodos provenientes de la ingeniería kansei para determinar las emociones que generan estas especies y su relación con la textura visual, patrón decorativo, color superficial, brillo y presencia de nudos. El estudio busca generar información útil a los proyectistas que tienen por responsabilidad realizar 
elecciones referidas a los materiales visualmente expuestos en el hábitat, aportando hacia el diseño de espacios con mejor proyección de aceptación usuaria.

\section{MATERIALES Y MÉTODOS}

El planteamiento metodológico se definió para determinar el nivel de aceptación emocional usuaria referido a la madera de cinco especies comerciales de Chile. El procedimiento de análisis estadístico consideró las frecuencias de respuestas, proporcionando resultados útiles para organizar los valores en orden jerárquico y realizar conclusiones en relación con la calidad percibida de las cinco maderas sometidas a estudio. Se efectuó un análisis de regresión lineal múltiple con la finalidad de evaluar la relación y el efecto de las variables que componen las cualidades superficiales de las maderas, con las emociones que influyen en la preferencia. En las tablas 1 y 2, se asigna 1 para el punto de menor preferencia, 4 al de más alta aceptación y 0 para neutralidad de acuerdo con la posición escalar.

TABLA 1. Diferencial semántico aplicado en una tabla con escala Likert para conocer las emociones que provoca en los entrevistados la madera de una especie.

\begin{tabular}{|c|c|c|c|c|c|}
\hline & $\begin{array}{c}\text { Totalmente } \\
4\end{array}$ & $\begin{array}{c}\text { En parte } \\
3\end{array}$ & $\begin{array}{c}\text { Neutro } \\
0\end{array}$ & $\begin{array}{c}\text { En parte } \\
2\end{array}$ & $\begin{array}{c}\text { Totalmente } \\
1\end{array}$ \\
\hline Ecológico & & & & & Artificial \\
\hline Acogedor & & & & & Inhóspito \\
\hline Atractivo & & & & & Repelente \\
\hline Jerarquía & & & & & Sencillo \\
\hline Calidez & & & & & Frialdad \\
\hline Robustez & & & & & Fragilidad \\
\hline
\end{tabular}

TABLA 2. Para recoger antecedentes para determinar la calidad percibida de los tipos de madera seleccionados empleando escala Likert.

\begin{tabular}{|c|c|c|c|c|c|c|}
\hline Prec & unta 2: De las siguie & ualidade & rficiales, cuc & ssideró p & su valo & \\
\hline & & & 5 el nivel de & cia en s & oració & \\
\hline & Elemento & $\begin{array}{c}\text { Mucho } \\
3\end{array}$ & $\begin{array}{c}\text { Suficiente } \\
2\end{array}$ & $\begin{array}{c}\text { Neutro } \\
0\end{array}$ & $\begin{array}{c}\text { Poco } \\
1\end{array}$ & $\begin{array}{c}\text { Nada } \\
\mathrm{O}\end{array}$ \\
\hline 1 & Textura visual & & & & & \\
\hline 2 & Color superficial & & & & & \\
\hline 3 & Brillo & & & & & \\
\hline 4 & Presencia nudos & & & & & \\
\hline 5 & Patrón decorativo & & & & & \\
\hline 6 & Tonalidad oscura & & & & & \\
\hline 7 & Tonalidad clara & & & & & \\
\hline
\end{tabular}


Determinación de las especies de maderas a estudiar

Para determinar las especies a estudiar se realizó una revisión bibliográfica y un catastro de las distribuidoras de madera en Chile, mediante análisis de directorios de ventas comerciales. En el primer caso se pudo constatar que las especies forestales comerciales nativas que se sitúan dentro de las más vendidas son: raulí (Nothofagus alpina (Poepp. y Endl.) Oerst.), coigüe (Nothofagus dombeyi (Mirb.) Oerst.), lenga (Nothofaguspumilio (Poepp. $y$ Endl.) Krasser.), roble (Nothofagus obliqua (Mirb.) Oerst. y mañío (Podocarpus nubigenus Lindl.). Por otra parte, las especies exóticas corresponden a aquellas originarias de otros países e introducidas en Chile, destacando entre ellas el pino radiata (Pinus radiata D. Don), debido a que corresponde a la especie mayormente plantada y vendida en el país (DíazVaz, Cuevas, Fernández e Inzunza, 2012; Huber y Trecaman, 2004). Para el segundo caso, se seleccionaron diez empresas y se efectuó una encuesta presencial entre la región Metropolitana y la región de la Araucanía, para determinar cuáles habían sido las maderas más vendidas en el periodo septiembre de 2015 a igual mes de 2016, caso en que se definió que las especies correspondientes son: raulí (N. alpina), pino radiata (P. radiata), roble (N. obliqua), pino Oregón (Pseudotsuga menžiesii (Mirb.) Franco), Eucalyptus nitens (Dean. et Maiden.). Acorde con la información recogida, se determinó que las maderas a considerar en la investigación serían las más vendidas en Chile en el periodo consultado, priorizando el criterio relativo a la preferencia del mercado nacional.

\section{Parámetros para la recogida de datos}

a) Instrumentos para la recogida de datos. Se aplicó un instrumento de recogida de datos con tablas definidas por diferencial semántico, basado en el sistema de evaluación psicológica (Demir, Desmet y Hekkert, 2009), que plantea que un concepto adquiere significado cuando un signo -palabra- puede provocar una respuesta asociada con el objeto que representa. En el marco de esta investigación, el objeto es una muestra de $500 \mathrm{~mm} \times 500$ $\mathrm{mm}$ de cada una de las cinco maderas en estudio, complementado con una imagen de ambiente con aplicaciones de madera empleando las mismas proporciones y formato. El instrumento consistió en un cuestionario de opciones cuantificables con un apartado que incluye datos demográficos de los entrevistados y preguntas estructuradas acorde con ejes semánticos asociados a emociones posibles de provocar por la madera y características definidas por sus cualidades superficiales. Quedó constituido principalmente por dos tablas y sus respectivas preguntas:

b) Procedimiento muestral. Se consideró una muestra no probabilística intencionada, seleccionando a expertos del área arquitectura, diseño y construcción, que por su condición de proyectistas constituían un perfil adecuado para esta investigación de carácter exploratorio. Participaron 9 mujeres $(37,5 \%)$ y 15 varones $(62,5 \%)$, seleccionados por muestreo no probabilístico por conveniencia. El promedio de edad de los encuestados es de 37 años (D.E. = 15,9; entre 21 años y 78 años). Este método de selección permitió procurar percepciones confiables, asegurando una perspectiva técnica-emocional, aunque la muestra fuera aleatoria.

\section{c) Metodología aplicada al desarrollo del grupo} focal. La experiencia se realizó en una sala donde predominó el color blanco tanto en paredes como en el equipamiento, para proporcionar un ambiente neutro. El procedimiento para realizar el grupo focal quedó definido en las siguientes etapas: se dio la bienvenida a los entrevistados y presentaron los objetivos de la investigación; se entregó el cuestionario y los entrevistados respondieron las preguntas en la medida que se presentaron una a una las diez modelaciones $2 \mathrm{D}$ (fotomontajes) que se diseñaron en color y dos para cada especie, acompañada de una muestra de madera sólida que se presentó de manera simultánea a la imagen gráfica. Se empleó un equipo Data show de alta definición para proyectar en un tiempo de exposición que se definió en 30 segundos para cada fotomontaje (Fig. 1, Fig. 2); por último, se procedió a recuperar los cuestionarios respondidos y entablar un diálogo con los asistentes sobre la experiencia realizada y la 
calidad percibida (Sakuragawa, S., Miyazaki, Kaneco y Makita, 2005).

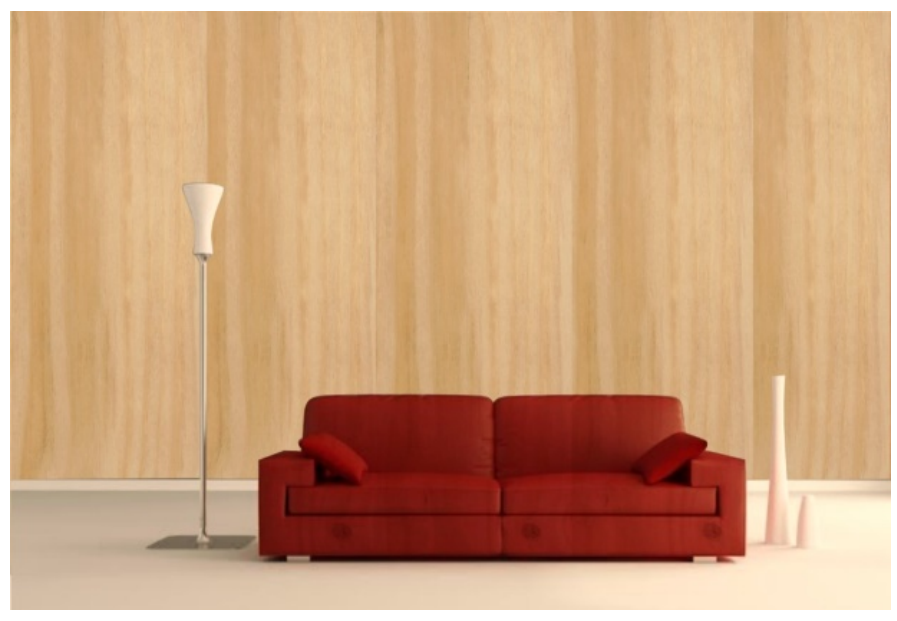

FIGURA 1. Fotomontaje Eucalyptus nitens (Dean. et Maiden.). Fuente: elaboración propia

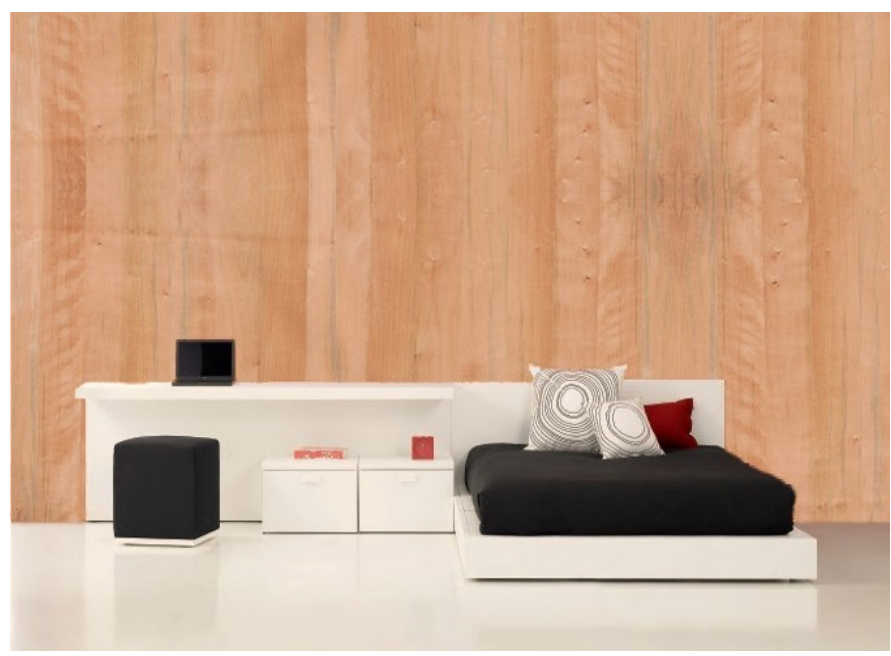

FIGURA 2. Fotomontaje raulí (N. alpina).

Fuente: elaboración propia

d) Tratamiento de datos. Se realizaron procedimientos estadísticos de análisis descriptivos, valoración global, frecuencias y determinación del orden de importancia y perfiles semánticos. El análisis descriptivo consistió en una depuración y control de datos, distribución de medias, frecuencias, varianza, dispersión y desviación típica. Se agruparon los resultados y gráficos, considerados como parte de los análisis regulares. Se realizó una valoración global de los componentes asimilados como variables, se analizó su recurrencia. Los datos se ordenan usando como patrón las frecuencias y correlaciones bivariadas para medir el grado de asociación entre ellas, cuantificadas en los coeficientes de Pearson.

\section{Resultados}

En las tablas 3 a 7 se muestran los resultados obtenidos para cada tipo de madera, respecto a los ejes semánticos referidos a la emoción y calidad percibida, en relación con sus cualidades superficiales.

Se aprecian diferencias entre las medias de las emociones, referidas a los elementos que conforman las cualidades superficiales de la madera y entre la valoración de preferencia de éstas. La tabla 8 presenta un resumen de los valores obtenidos por las variables emocionales, ponderados según las frecuencias obtenidas en las escalas de valoración y que permiten organizar los tipos de especies en orden de preferencia. Se incorpora el porcentaje para una lectura más completa de los resultados.

En la tabla 8 se considera a $N$. alpina como la madera mejor valorada $(\Sigma=490)$ con $26,16 \%$ de la preferencias, dejando a la madera de $N$. obliqua con 20,13\% en segundo lugar $(\Sigma=377)$, después $P$. menziesii $(\Sigma=362)$ con $19,33 \%$, E. nitens con $18,37 \%(\Sigma=344) \mathrm{y}$, finalmente, Pinus radiata con 16,02\% $(\Sigma=300)$. En el eje atractivo-repelente también supera este promedio $(\Sigma=72)$, a pesar que es sobrepasado en esta dimensión por la madera de $N$. obliqua $(\Sigma=73)$. $N$. obliqua, segunda en elegibilidad, destaca por ser percibida como acogedora $(\Sigma=72)$, atractiva $(\Sigma=73)$, cálida $(\Sigma=$ 74) y ecológica $(\Sigma=64)$, pero a juicio de los entrevistados carece de jerarquía $(\Sigma=35)$, situándola entre P. menziesii y Pinus radiata. Para la madera de $P$. menziesii la sensación de acogedor $(\Sigma=70)$, atractivo $(\Sigma=66)$ y cálido $(\Sigma=66)$, supera el promedio de aceptación, pero es débil en robustez $(\Sigma=58)$ y jerarquía $(\Sigma=38)$. Con menor orden de preferencia, E. nitens sobresale levemente en ser atractivo ( $\Sigma=68)$ y ecológico $(\Sigma=68)$, apenas por sobre el promedio. Pinus radiata, ocupa el último lugar para este orden, ya que el promedio de los ejes sobrepasa ligeramente su condición ecológica $(\Sigma=66)$, quedando los demás aspectos disminuidos en significación estadística. 
TABLA 3. Estadísticos descriptivos de las variables para N. obliqua.

\begin{tabular}{llcccc}
\hline \multicolumn{1}{c}{ N. obliqua } & & Media & D.E. & $s^{2}$ & $\Sigma$ \\
\hline & Ecológico - artificial & 2,56 & 1,50 & 2,26 & 64 \\
& Acogedor - inhóspito & 2,88 & 1,39 & 1,94 & 72 \\
Emociones & Atractivo - repelente & 2,92 & 1,26 & 1,58 & 73 \\
Ejes semánticos & Jerarquía - sencillo & 1,40 & 1,47 & 2,17 & 35 \\
& Calidez - frialdad & 2,96 & 1,34 & 1,79 & 74 \\
& Robustez - fragilidad & 2,36 & 1,55 & 2,41 & 59 \\
\hline \multirow{4}{*}{$\begin{array}{l}\text { Cualidades } \\
\text { superficiales de }\end{array}$} & Textura visual & 2,16 & 1,03 & 1,06 & 54 \\
madera & Color superficial & 2,36 & 0,81 & 0,66 & 59 \\
& Prillo & 1,32 & 1,07 & 1,14 & 33 \\
& Patrón decorativo & 2,12 & 1,09 & 1,19 & 53 \\
& Tonalidad oscura & 1,40 & 1,22 & 1,50 & 35 \\
& Tonalidad clara & 1,60 & 1,08 & 1,17 & 40 \\
\hline
\end{tabular}

D.E: Desviación Estándar

$\mathrm{S}^{2}$ : Varianza

$\Sigma:$ Suma

TABLA 4. Estadísticos descriptivos de las variables para Pinus radiata.

\begin{tabular}{llcccc}
\hline Pinus radiata & & Media & D.E. & $s^{2}$ & $\Sigma$ \\
\hline & Ecológico - artificial & 2,64 & 1,44 & 2,07 & 66 \\
& Acogedor - inhóspito & 2,40 & 1,15 & 1,33 & 60 \\
Emociones & Atractivo - repelente & 1,92 & 1,44 & 2,08 & 48 \\
Ejes & Jerarquía - sencillo & 1,24 & 1,48 & 2,19 & 31 \\
semánticos & Calidez - frialdad & 2,20 & 1,41 & 2,00 & 55 \\
& Robustez - fragilidad & 1,60 & 1,41 & 2,00 & 40 \\
\hline \multirow{4}{*}{ Cualidades } & Textura visual & 2,20 & 0,87 & 0,75 & 55 \\
superficiales & Prillo & 2,16 & 0,90 & 0,81 & 54 \\
de la madera & Presencia nudos & 1,44 & 1,16 & 1,34 & 36 \\
& Patrón decorativo & 1,40 & 1,32 & 1,75 & 35 \\
& Tonalidad oscura & 1,76 & 1,09 & 1,19 & 44 \\
& Tonalidad clara & 1,64 & 0,91 & 0,82 & 41 \\
& Color superficial & 1,92 & 1,08 & 1,16 & 48 \\
\hline
\end{tabular}

D.E: Desviación Estándar

$S^{2}$ : Varianza

$\sum$ : Suma 
TABLA 5. Estadísticos descriptivos de las variables para P. menziesii.

\begin{tabular}{llcccc}
\hline \multicolumn{1}{c}{ P. menziesii } & & Media & D.E. & $s^{2}$ & $\Sigma$ \\
\hline & Ecológico - artificial & 2,56 & 1,47 & 2,17 & 64 \\
& Acogedor - inhóspito & 2,80 & 1,32 & 1,75 & 70 \\
Emociones & Atractivo - repelente & 2,64 & 1,29 & 1,66 & 66 \\
Ejes semánticos & Jerarquía - sencillo & 1,52 & 1,50 & 2,26 & 38 \\
& Calidez - frialdad & 2,64 & 1,41 & 1,99 & 66 \\
& Robustez - fragilidad & 2,32 & 1,52 & 2,31 & 58 \\
\hline \multirow{4}{*}{ Cualidades } & Textura visual & 2,32 & 0,90 & 0,81 & 58 \\
superficiales de la & Presencia nudos & 1,60 & 1,19 & 1,42 & 40 \\
madera & Color superficial & 2,08 & 1,08 & 1,16 & 52 \\
& Patrón decorativo & 2,00 & 1,15 & 1,33 & 50 \\
& Tonalidad oscura & 1,12 & 1,17 & 1,36 & 28 \\
& Tonalidad clara & 1,28 & 1,28 & 1,63 & 32 \\
\hline
\end{tabular}

D.E: Desviación Estándar

$\mathrm{S}^{2}$ : Varianza

$\sum$ : Suma

TABLA 6. Estadísticos descriptivos de las variables para E. nitens.

\begin{tabular}{llcccc}
\hline \multicolumn{1}{c}{ E. nitens } & & Media & D.E. & $s^{2}$ & $\Sigma$ \\
\hline & Ecológico - artificial & 2,72 & 1,49 & 2,21 & 68 \\
& Acogedor - inhóspito & 2,20 & 1,41 & 2,00 & 55 \\
Emociones & Atractivo - repelente & 2,72 & 1,24 & 1,54 & 68 \\
Ejes semánticos & Jerarquía - sencillo & 1,96 & 1,46 & 2,12 & 49 \\
& Calidez - frialdad & 1,96 & 1,27 & 1,62 & 49 \\
& Robustez - fragilidad & 2,20 & 1,55 & 2,42 & 55 \\
\hline \multirow{4}{*}{ Cualidades } & Textura visual & 2,28 & 0,84 & 0,71 & 57 \\
superficiales de & Color superficial & 2,12 & 1,09 & 1,19 & 53 \\
la madera & Presencia nudos & 1,28 & 1,17 & 1,38 & 32 \\
& Patrón decorativo & 1,96 & 1,23 & 1,52 & 31 \\
& Tonalidad oscura & 0,80 & 1,08 & 1,04 & 49 \\
& Tonalidad clara & 1,84 & 1,14 & 1,31 & 46 \\
\hline
\end{tabular}

D.E: Desviación Estándar

$\mathrm{S}^{2}$ : Varianza

$\Sigma$ : Suma 
TABLA 7. Estadísticos descriptivos de las variables para N. alpina.

\begin{tabular}{llcccc}
\hline \multicolumn{1}{c}{ N. alpina } & & Media & D.E. & $s^{2}$ & $\Sigma$ \\
\hline & Ecológico - artificial & 3,36 & 1,15 & 1,32 & 84 \\
& Acogedor - inhóspito & 3,48 & 0,87 & 0,76 & 87 \\
Emociones & Atractivo - repelente & 2,88 & 1,54 & 2,36 & 72 \\
Ejes semánticos & Jerarquía - sencillo & 3,12 & 1,27 & 1,61 & 78 \\
& Calidez - frialdad & 3,56 & 0,51 & 0,26 & 89 \\
& Robustez - fragilidad & 3,20 & 1,29 & 1,67 & 80 \\
\hline \multirow{4}{*}{ Cualidades } & Textura visual & 2,76 & 0,44 & 0,19 & 69 \\
superficiales de la & Presencia nudos & 2,04 & 1,06 & 1,12 & 51 \\
madera & Color superficial & 2,56 & 0,71 & 0,51 & 64 \\
& Patrón decorativo & 2,52 & 0,71 & 0,51 & 63 \\
& Tonalidad oscura & 2,32 & 0,90 & 0,81 & 58 \\
& Tonalidad clara & 1,08 & 1,12 & 1,24 & 27 \\
\hline
\end{tabular}

D.E: Desviación Estándar

$S^{2}$ : Varianza

$\sum$ : Suma

TABLA 8. Orden de preferencia según emociones respecto de los ejes semánticos en las cualidades superficiales de las especies en estudio.

\begin{tabular}{lcccccccc}
\hline & $\begin{array}{c}\text { Ecológico } \\
\text { Artificial }\end{array}$ & $\begin{array}{c}\text { Acogedor } \\
\text { Inhóspito }\end{array}$ & $\begin{array}{c}\text { Atractivo } \\
\text { Repelente }\end{array}$ & $\begin{array}{c}\text { Jerarquía } \\
\text { Sencillo }\end{array}$ & $\begin{array}{c}\text { Calidez } \\
\text { Frialdad }\end{array}$ & $\begin{array}{c}\text { Robustez } \\
\text { Fragilidad }\end{array}$ & $\begin{array}{c}\text { Suma } \\
\text { Preferencias }\end{array}$ & $\begin{array}{c}\text { Porcentaje } \\
\text { (\%) }\end{array}$ \\
\hline N. obliqua & 64 & 72 & 73 & 35 & 74 & 59 & 377 & 20,13 \\
Pinus radiata & 66 & 60 & 48 & 31 & 55 & 40 & 300 & 16,06 \\
P. menziesii & 64 & 70 & 66 & 38 & 66 & 58 & 362 & 19,33 \\
E. nitens & 68 & 55 & 68 & 49 & 49 & 55 & 344 & 18,37 \\
N. alpina & 84 & 87 & 72 & 78 & 89 & 80 & 490 & 26,16 \\
\hline
\end{tabular}

La diferencia en la ponderación obtenida por cada muestra de madera presentada, está relacionada con elementos que componen las cualidades superficiales que las distinguen entre ellas. Es así que la madera de N. alpina destaca por la ponderación alcanzada sobre las otras maderas en la cualidades superficiales $(\Sigma=69)$, color superficial $(\Sigma=64)$, presencia de nudos $(\Sigma=51)$, patrón decorativo $(\Sigma=63)$ y tonalidad oscura $(\Sigma=58)$, según se aprecia en la tabla 9; sin embargo, no destaca por el brillo superficial $(\Sigma=25) \mathrm{ni}$ tampoco por la tonalidad clara $(\Sigma=27)$, apareciendo como cualidades desmejoradas. Dentro de las características de $N$. obliqua, se encuentra el color superficial $(\Sigma=59)$ y el patrón decorativo $(\Sigma=53)$. Por su parte, E. nitens destaca por su textura visual $(\Sigma=57)$, color superficial $(\Sigma=53)$ y patrón decorativo $(\Sigma=49)$. El brillo destaca a Pinus radiata $(\Sigma=$ 36) por sobre las demás maderas, aunque esté situado al final de las preferencias. El P. menziesii, que mantiene alto nivel de preferencias en textura visual $(\Sigma=58)$, en la particularidad de sus nudos $(\Sigma=40)$ y en patrón decorativo $(\Sigma=50)$, no destaca en otros elementos visuales en su superficie. 
TABLA 9. Cualidades superficiales de las maderas, ponderadas según frecuencia en marcas de preferencia.

\begin{tabular}{lccccccccc}
\hline & $\begin{array}{c}\text { Textura } \\
\text { visual }\end{array}$ & $\begin{array}{c}\text { Color } \\
\text { superficial }\end{array}$ & Brillo & $\begin{array}{c}\text { Presencia } \\
\text { nudos }\end{array}$ & $\begin{array}{c}\text { Patrón } \\
\text { decorativo }\end{array}$ & $\begin{array}{c}\text { Tonalidad } \\
\text { oscura }\end{array}$ & $\begin{array}{c}\text { Tonalidad } \\
\text { clara }\end{array}$ & $\begin{array}{c}\text { Suma } \\
\text { Preferencias }\end{array}$ & $\begin{array}{c}\text { Porcentaje } \\
\text { (\%) }\end{array}$ \\
\hline $\begin{array}{l}\text { N. obliqua } \\
\text { Pinus }\end{array}$ & 54 & 59 & 33 & 39 & 53 & 35 & 40 & 313 & 20,06 \\
$\begin{array}{l}\text { radiata } \\
\text { P. }\end{array}$ & 55 & 54 & 36 & 35 & 44 & 41 & 48 & 313 & 20,06 \\
menziesii & 58 & 52 & 29 & 40 & 50 & 28 & 32 & 289 & 18,53 \\
E. nitens & 57 & 53 & 32 & 31 & 49 & 20 & 46 & 288 & 18,46 \\
N. alpina & 69 & 64 & 25 & 51 & 63 & 58 & 27 & 357 & 22,88 \\
\hline
\end{tabular}

La madera de N. alpina expresa características que la diferencian por sobre otras y que la hacen favorita (Tabla 10). Existe una alta correlación entre acogedor y su color superficial $(r=0,82 \mathrm{p}<0,01)$, mientras que la presencia de nudos $(r=0,52 p<0,01)$ y su tonalidad oscura $(r=0,65 \mathrm{p}$ $<0,01)$ correlacionan moderadamente con acogedor, contribuyendo a fortalecer la sensación de confort. Su color superficial correlaciona medianamente $(r=0,44 \mathrm{p}<0,05)$ con atractivo, al igual que el brillo $(r=0,46 \mathrm{p}<0,05)$ y la presencia de nudos $(r=0,64 \mathrm{p}<0,05)$. La sensación de calidez se relaciona moderadamente con el patrón decorativo $(r=0,54 \mathrm{p}<0,01)$, lo mismo que la percepción de robustez lo hace con el color superficial $(r=0,55 \mathrm{p}<$ $0,01)$ y su tonalidad oscura $(r=0,41 \mathrm{p}<0,05)$.

En la tabla 11, se exponen las correlaciones entre las emociones y los elementos que componen las cualidades superficiales de $N$. obliqua. Existe una correlación media y significativa entre ecológico y textura visual para la madera de $N$. obliqua $(r=0,40 \mathrm{p}<0,05)$. La presencia de nudos y el patrón decorativo ofrecen una sensación placentera y grata de acogida, según se interpreta por la correlación moderada y significativa entre estos componentes $(r=0,59 \mathrm{p}<0,01 ; r$ $=0,50 \mathrm{p}<0,05$ respectivamente). Lo atractivo correlaciona significativamente con el color superficial de moderado a alto, $(\mathrm{r}=0,68 \mathrm{p}<0,01)$. La condición de robustez en $N$. obliqua, está correlacionada ampliamente con la textura visual $(\mathrm{r}=0,62 \mathrm{p}<0,01)$ y su tonalidad oscura $(\mathrm{r}=0,64 \mathrm{p}$ $<0,01)$ y, solo medianamente, con la presencia de nudos ( $\mathrm{r}$ $=0,47 \mathrm{p}<0,05)$.
Las preferencias obtenidas por la madera de P. menziesii (Tabla 12), correlacionan moderadamente su atractivo con la textura visual $(r=0,50 p<0,05) \mathrm{y}$, en mejor significancia, con su color superficial $(r=0,62 p<0,01)$. También destaca la correlación media inversa entre el aspecto ecológico que presenta y el brillo superficial. La sensación de calidez se relaciona medianamente con su textura visual $(r=0,46 p<0,05)$. Menor preferencia tiene E. nitens (Tabla 13), ya que solo dos ejes de las escalas emocionales presentan correlaciones significativas. Su atractivo muestra relación media e inversa con su tonalidad oscura $(r=$ $0,45 p<0,05)$ y su cualidad de robustez establece correlación media con el patrón decorativo $(r=0,40 p<0,05)$. Para Pinus radiata (Tabla 14), solo aparece como correlación media y significativa lo ecológico $(r=0,52 p<0,01)$. Los demás elementos que componen sus cualidades superficiales no se destacan.

El modelo de regresión respecto a los ejes semánticos para la madera de $N$. alpina obtuvo $\mathrm{R}^{2}$ de $0,808\left(\mathrm{R}^{2}\right.$ adj $=$ $0,729)$, coeficientes significativamente adecuados $\left(\mathrm{F}_{7,17}=\right.$ $10,241, \mathrm{p}<0,001)$, indicando que existe una relación lineal directa entre el color superficial y la presencia de nudos, como predictores de la condición de material esencialmente acogedora. Del mismo modo, se estudiaron los coeficientes para el eje atractivo-repelente obteniéndose los valores de $\mathrm{R}^{2}$ de 0,468 $\left(\mathrm{R}^{2} \mathrm{adj}=0,420\right)$, con significación estadística $\left(\mathrm{F}_{2,22}=10,920, \mathrm{p}<0,01\right)$. Estos valores muestran que la presencia de nudos y el patrón decorativo hacen efectivamente más atractiva la madera de $N$. alpina. 
TABLA 10. Correlación de Pearson entre ejes emocionales y cualidades superficiales para N. alpina.

\begin{tabular}{|c|c|c|c|c|c|c|c|c|c|c|c|c|c|}
\hline N. alpina & 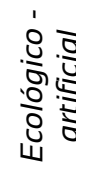 & 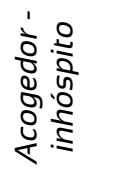 & 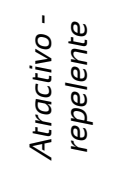 & 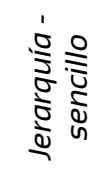 & 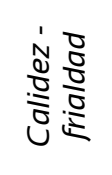 & 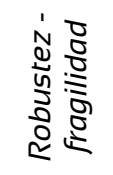 & 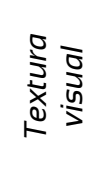 & 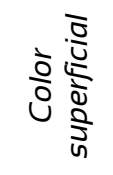 & $\stackrel{\circ}{\stackrel{0}{*}}$ & 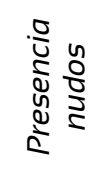 & 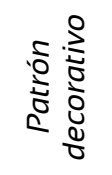 & 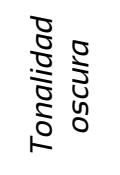 & $\begin{array}{l}\frac{1}{0} 0 \\
\frac{1}{0} \frac{0}{0} \\
\frac{0}{0} \\
0\end{array}$ \\
\hline Ecológico - artificial & & 0,11 & $-0,02$ & $0,45^{*}$ & 0,35 & 0,26 & 0,26 & 0,00 & 0,00 & 0,09 & 0,02 & $-0,04$ & 0,24 \\
\hline Acogedor - inhóspito & & & $0,57^{* *}$ & $-0,02$ & 0,31 & $0,73^{* *}$ & 0,21 & $0,82^{* *}$ & 0,24 & $0,52^{* *}$ & 0,12 & $0,65^{* *}$ & 0,30 \\
\hline Atractivo - repelente & & & & $-0,01$ & $0,46^{*}$ & 0,29 & 0,20 & $0,44^{*}$ & $0,46^{*}$ & $0,64^{* *}$ & 0,17 & 0,39 & 0,18 \\
\hline Jerarquía - sencillo & & & & & $0,54^{* *}$ & 0,16 & 0,13 & 0,11 & $-0,17$ & 0,00 & 0,07 & $-0,04$ & 0,05 \\
\hline Calidez - frialdad & & & & & & 0,27 & 0,26 & 0,37 & 0,14 & 0,34 & $0,54^{* *}$ & 0,32 & 0,21 \\
\hline Robustez - fragilidad & & & & & & & 0,39 & $0,55^{* *}$ & 0,11 & 0,33 & 0,02 & $0,41^{*}$ & 0,16 \\
\hline Textura visual & & & & & & & & 0,32 & 0,08 & 0,38 & 0,02 & 0,31 & $-0,30$ \\
\hline Color superficial & & & & & & & & & 0,25 & $0,52^{* *}$ & 0,30 & $0,68^{* *}$ & 0,15 \\
\hline Brillo & & & & & & & & & & $0,43^{*}$ & 0,29 & 0,23 & $0,53^{* *}$ \\
\hline Presencia nudos & & & & & & & & & & & $0,47^{*}$ & $0,42^{*}$ & 0,03 \\
\hline Patrón decorativo & & & & & & & & & & & & 0,25 & 0,10 \\
\hline Tonalidad oscura & & & & & & & & & & & & & 0,01 \\
\hline Tonalidad clara & & & & & & & & & & & & & \\
\hline
\end{tabular}

${ }^{*} p<0.05 .{ }^{* *} p<0.01$

TABLA 11. Correlación de Pearson entre ejes emocionales y componentes cualidades superficiales para N. obliqua.

\begin{tabular}{|c|c|c|c|c|c|c|c|c|c|c|c|c|c|}
\hline N. obliqua & 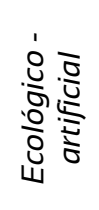 & 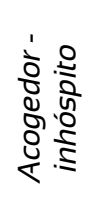 & 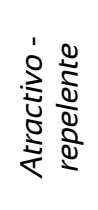 & 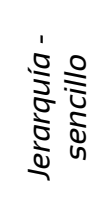 & 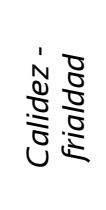 & 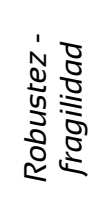 & 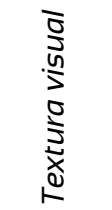 & 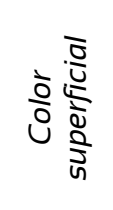 & 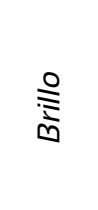 & 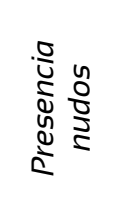 & 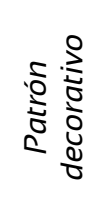 & 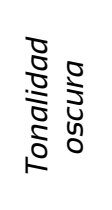 & $\begin{array}{l}\frac{1}{0} \\
\frac{1}{0} \\
\frac{0}{0} \\
\frac{0}{0} \\
0\end{array}$ \\
\hline Ecológico - artificial & & 0,29 & 0,33 & $-0,03$ & 0,05 & $0,48^{*}$ & $0,40^{*}$ & 0,31 & 0,30 & 0,14 & 0,31 & 0,35 & $-0,09$ \\
\hline Acogedor - inhóspito & & & 0,23 & $-0,08$ & $0,67^{* *}$ & 0,39 & $-0,02$ & 0,26 & 0,14 & $0,59^{* *}$ & $0,50^{*}$ & 0,27 & $-0,14$ \\
\hline Atractivo - repelente & & & & 0,04 & 0,22 & 0,19 & 0,33 & $0,68^{* *}$ & 0,11 & 0,11 & 0,22 & 0,37 & $-0,09$ \\
\hline Jerarquía - sencillo & & & & & $-0,18$ & $-0,12$ & $-0,07$ & 0,36 & 0,26 & 0,01 & 0,33 & $-0,16$ & 0,10 \\
\hline Calidez - frialdad & & & & & & 0,23 & $-0,06$ & 0,13 & $-0,14$ & $0,51^{* *}$ & 0,29 & 0,26 & 0,07 \\
\hline Robustez - fragilidad & & & & & & & $0,62^{* *}$ & 0,26 & 0,25 & $0,47^{*}$ & 0,34 & $0,64^{* *}$ & $-0,18$ \\
\hline Textura visual & & & & & & & & $0,58^{* *}$ & 0,29 & 0,26 & 0,20 & $0,44^{*}$ & $-0,05$ \\
\hline Color superficial & & & & & & & & & 0,29 & 0,13 & $0,61^{* *}$ & $0,44^{*}$ & 0,03 \\
\hline Brillo & & & & & & & & & & 0,25 & 0,22 & $0,50^{*}$ & $-0,14$ \\
\hline Presencia nudos & & & & & & & & & & & 0,30 & 0,27 & $-0,11$ \\
\hline Patrón decorativo & & & & & & & & & & & & $0,40^{*}$ & $-0,06$ \\
\hline Tonalidad oscura & & & & & & & & & & & & & 0,06 \\
\hline Tonalidad clara & & & & & & & & & & & & & \\
\hline
\end{tabular}

${ }^{*} \mathrm{P}<0.05 .{ }^{* *} \mathrm{P}<0.01$ 
TABLA 12. Correlación de Pearson entre ejes emocionales y componentes cualidades superficiales para P. menziesii.

\begin{tabular}{|c|c|c|c|c|c|c|c|c|c|c|c|c|c|}
\hline P. menziesii & 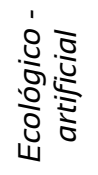 & 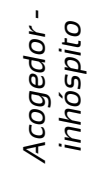 & 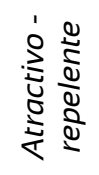 & 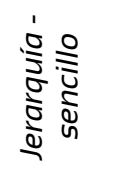 & ن & 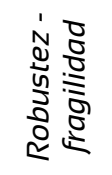 & 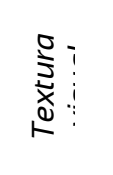 & 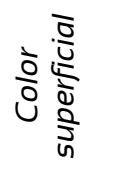 & 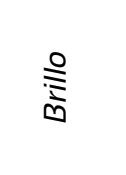 & 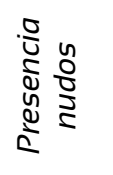 & 过 & 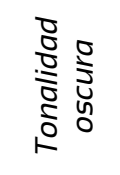 & $\begin{array}{l}\frac{5}{0} \\
\frac{0}{0} \frac{0}{0} \\
\frac{0}{0} \\
0\end{array}$ \\
\hline Ecológico - artificial & & 0,12 & 0,33 & $-0,06$ & 0,00 & 0,07 & 0,05 & 0,02 & $-0,49^{*}$ & $-0,22$ & 0,05 & $-0,06$ & $-0,18$ \\
\hline Acogedor - inhóspito & & & 0,32 & 0,05 & $0,56^{* *}$ & $0,57^{* *}$ & 0,27 & 0,13 & 0,17 & 0,26 & 0,08 & 0,39 & $-0,06$ \\
\hline Atractivo - repelente & & & & $0,40^{*}$ & 0,06 & $0,42^{*}$ & $0,50^{*}$ & $0,62^{* *}$ & 0,01 & 0,07 & 0,25 & 0,28 & 0,27 \\
\hline Jerarquía - sencillo & & & & & 0,19 & 0,31 & 0,27 & 0,28 & 0,39 & $-0,11$ & 0,07 & 0,34 & 0,07 \\
\hline Calidez - frialdad & & & & & & $0,58^{* *}$ & $0,46^{*}$ & $-0,01$ & 0,08 & 0,08 & 0,10 & 0,31 & $-0,08$ \\
\hline Robustez - fragilidad & & & & & & & 0,38 & 0,16 & 0,08 & 0,03 & 0,19 & 0,26 & $-0,05$ \\
\hline Textura visual & & & & & & & & $0,57^{\star *}$ & 0,13 & 0,12 & 0,36 & 0,16 & 0,21 \\
\hline Color superficial & & & & & & & & & 0,38 & 0,29 & $0,40^{*}$ & $0,46^{*}$ & $0,44^{*}$ \\
\hline Brillo & & & & & & & & & & 0,18 & 0,06 & $0,52^{\star *}$ & 0,30 \\
\hline Presencia nudos & & & & & & & & & & & $0,45^{*}$ & $0,40^{*}$ & 0,24 \\
\hline Patrón decorativo & & & & & & & & & & & & 0,37 & $0,51^{* *}$ \\
\hline Tonalidad oscura & & & & & & & & & & & & & 0,17 \\
\hline Tonalidad clara & & & & & & & & & & & & & \\
\hline
\end{tabular}

${ }^{*} \mathrm{P}<0.05 .{ }^{* *} \mathrm{P}<0.01$

TABLA 13. Correlación de Pearson entre ejes emocionales y componentes cualidades superficiales para E. nitens.

\begin{tabular}{|c|c|c|c|c|c|c|c|c|c|c|c|c|c|}
\hline E. nitens & 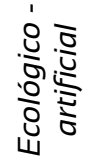 & 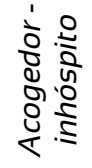 & 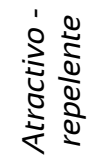 & 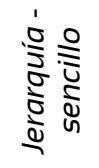 & 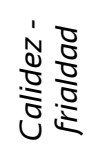 & 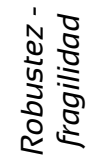 & 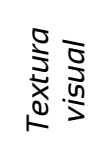 & 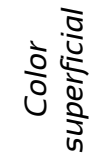 & $\stackrel{\circ}{\stackrel{0}{*}}$ & 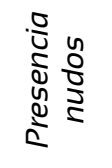 & 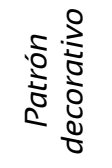 & $\begin{array}{l}0 \\
0 \\
0 \\
0 \\
0 \\
0 \\
0 \\
0 \\
0 \\
0 \\
0\end{array}$ & $\begin{array}{l}\frac{1}{0} \\
\frac{0}{0} \\
\frac{0}{0} \\
\frac{0}{0} \\
0\end{array}$ \\
\hline Ecológico - artificial & & 0,36 & 0,05 & $-0,01$ & 0,10 & 0,30 & $-0,10$ & $-0,11$ & $-0,02$ & 0,22 & 0,38 & 0,28 & $-0,17$ \\
\hline Acogedor - inhóspito & & & 0,10 & $0,41^{*}$ & 0,07 & $0,72^{* *}$ & 0,16 & 0,25 & 0,14 & 0,21 & 0,32 & 0,08 & $-0,13$ \\
\hline Atractivo - repelente & & & & 0,22 & 0,31 & 0,27 & $-0,12$ & $-0,25$ & $-0,23$ & $-0,01$ & $-0,17$ & $-0,45^{*}$ & 0,08 \\
\hline Jerarquía - sencillo & & & & & 0,00 & 0,32 & 0,15 & 0,26 & 0,03 & 0,12 & $-0,03$ & $-0,38$ & $-0,08$ \\
\hline Calidez - frialdad & & & & & & 0,07 & $-0,30$ & 0,09 & 0,26 & $-0,36$ & $-0,07$ & $-0,07$ & 0,02 \\
\hline Robustez - fragilidad & & & & & & & 0,05 & 0,08 & $-0,03$ & 0,32 & $0,40^{*}$ & 0,15 & 0,04 \\
\hline Textura visual & & & & & & & & 0,32 & 0,25 & 0,01 & 0,11 & $-0,16$ & 0,22 \\
\hline Color superficial & & & & & & & & & $0,43^{*}$ & $-0,08$ & 0,19 & 0,16 & 0,22 \\
\hline Brillo & & & & & & & & & & $-0,48^{*}$ & $-0,03$ & 0,11 & 0,35 \\
\hline Presencia nudos & & & & & & & & & & & $0,40^{*}$ & 0,38 & 0,00 \\
\hline Patrón decorativo & & & & & & & & & & & & 0,30 & $-0,11$ \\
\hline Tonalidad oscura & & & & & & & & & & & & & 0,31 \\
\hline Tonalidad clara & & & & & & & & & & & & & \\
\hline
\end{tabular}

${ }^{*} \mathrm{P}<0.05 .{ }^{* *} \mathrm{P}<0.01$ 
TABLA 14. Correlación de Pearson entre ejes emocionales y componentes cualidades superficiales para Pinus radiata.

\begin{tabular}{|c|c|c|c|c|c|c|c|c|c|c|c|c|c|}
\hline Pinus radiata & 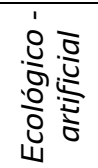 & 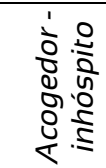 & 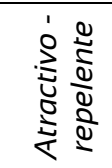 & 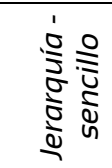 & 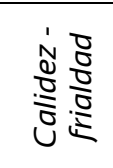 & 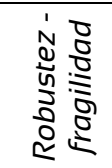 & 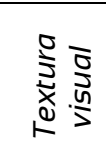 & 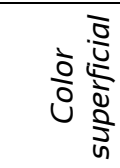 & $\stackrel{0}{\stackrel{0}{(0)}}$ & 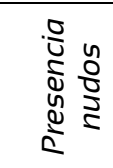 & 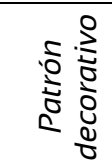 & 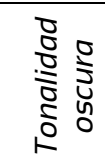 & 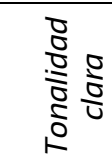 \\
\hline Ecológico - artificial & & 0,27 & 0,07 & $-0,31$ & 0,22 & 0,25 & $-0,01$ & $-0,05$ & $-0,28$ & $0,52^{* *}$ & 0,10 & $-0,01$ & 0,22 \\
\hline Acogedor - inhóspito & & & 0,20 & $-0,28$ & $0,43^{*}$ & 0,28 & 0,04 & 0,14 & $-0,23$ & 0,14 & 0,15 & $-0,14$ & 0,13 \\
\hline Atractivo - repelente & & & & $-0,40^{*}$ & $-0,13$ & $-0,18$ & 0,05 & 0,20 & 0,20 & 0,10 & 0,09 & 0,20 & 0,34 \\
\hline Jerarquía - sencillo & & & & & 0,00 & 0,09 & 0,19 & 0,10 & $-0,11$ & $-0,29$ & $-0,20$ & $-0,37$ & $-0,17$ \\
\hline Calidez - frialdad & & & & & & 0,35 & 0,10 & 0,17 & 0,02 & 0,24 & 0,17 & 0,38 & $-0,26$ \\
\hline Robustez - fragilidad & & & & & & & 0,27 & $-0,01$ & $-0,19$ & 0,20 & 0,23 & 0,05 & $-0,16$ \\
\hline Textura visual & & & & & & & & $0,60^{* *}$ & $-0,34$ & 0,11 & $0,45^{*}$ & 0,31 & $-0,25$ \\
\hline Color superficial & & & & & & & & & $-0,27$ & 0,01 & 0,21 & 0,18 & 0,10 \\
\hline Brillo & & & & & & & & & & 0,02 & $-0,14$ & 0,16 & 0,26 \\
\hline Presencia nudos & & & & & & & & & & & $0,50^{*}$ & 0,06 & 0,20 \\
\hline Patrón decorativo & & & & & & & & & & & & 0,29 & 0,20 \\
\hline Tonalidad oscura & & & & & & & & & & & & & $-0,33$ \\
\hline Tonalidad clara & & & & & & & & & & & & & \\
\hline
\end{tabular}

${ }^{*} \mathrm{P}<0.05 .{ }^{* *} \mathrm{P}<0.01$

En el caso de N. obliqua, para el eje ecológico-artificial, existe un nivel de predicción estadísticamente significativo con la textura visual, con coeficientes bajos cuyo valor es: $\mathrm{R}^{2}=0,159\left(\mathrm{R}^{2}\right.$ adj $\left.=0,122\right)$, con significación estadística $\left(\mathrm{F}_{1,23}=4,336, \mathrm{p}<0,05\right)$. Esto quiere decir que el aspecto ecológico que presenta $N$. obliqua se puede atribuir a la textura visual de la madera, ciertamente en un bajo porcentaje, pero igualmente significativo. Para el eje acogedor-inhóspito, es significativo y con moderado coeficiente de predicción que tanto la presencia de nudos como la del patrón decorativo influyen en la percepción de acogedor, entendido como agradable, cómodo, que brinda tranquilidad. El coeficiente estadístico es $\mathrm{R}^{2}=0,468\left(\mathrm{R}^{2}\right.$ adj $=0,420)$, de significación $\left.\left(\mathrm{F}_{1,23}=9,688, \mathrm{p}<0,001\right)\right)$. Del mismo modo se puede considerar que el color superficial es el que otorga características para ser considerada una madera atractiva, ya que posee coeficientes $\mathrm{R}^{2}$ de 0,469 $\left(\mathrm{R}^{2}\right.$ adj $\left.=0,446\right)$ con buena significación estadística $\left(\mathrm{F}_{2,22}=\right.$ 20,296, p <0,001). En cuanto a su robustez, el coeficiente $\mathrm{R}^{2}$ de $0,551\left(\mathrm{R}^{2}\right.$ adj $\left.=0,510\right)$ con significación estadística
$\mathrm{F}_{2,22}=13,497, \mathrm{p}<0,001$, indica que su robustez es atribuida a su tonalidad oscura y a la textura visual.

\section{DISCUSIÓN}

Las cualidades superficiales de la madera percibidas por los individuos, provocan distintas reacciones emocionales que influyen en el grado de preferencias de los encuestados; en otras palabras, correlacionan las categorías emocionales con la textura visual, patrón decorativo, color superficial, brillo y presencia de nudos. En esta medida, los valores recogidos en las escalas que conforman los ejes semánticos como respuestas emocionales frente a la exposición de las distintas maderas en estudio, están asociados a las características de cada especie y, como tal, su ponderación entrega valoraciones que pueden ser relevantes al momento de definir las maderas que estarán más expuestas a la vista en un determinado hábitat. A partir de este estudio se podría realizar una lista de recomendaciones, ya que efectivamente permite entregar una herramienta de valoración emocional a los proyectistas, los que podrán 
incorporar esta perspectiva en las decisiones asociadas al confort psicológico de los futuros usuarios de un espacio habitable, midiendo más cautelosamente la capacidad expresiva y comunicativa de las maderas que los conforman.

Las cualidades superficiales de la madera son determinantes al momento de jerarquizar las preferencias de los usuarios por un tipo de madera u otro. Los cinco aspectos considerados para este estudio, que se han mencionado anteriormente, permiten establecer preferencias de los entrevistados y su relación entre la calidad percibida y las emociones provocadas. Por ejemplo, la madera de N. alpina es valorada con altas ponderaciones por los entrevistados para los aspectos consultados, situándose en la categoría de mayor elegibilidad. Esta madera de color oscuro y homogéneo, con vetas tenues y baja presencia de nudos, se contrapone diametralmente con las cualidades superficiales de la madera de Pinus radiata, con vetas más marcadas y presencia frecuente de nudos que destacan por su tonalidad oscura que contrastan con la predominancia de color claro que la caracteriza. Ambas maderas, situadas en los extremos de preferencia, indican claramente que el valor emocional positivo de la madera se acerca a la tipología de madera de N. alpina.

Las cualidades superficiales de la madera correlacionan afectivamente evocando propiedades y rasgos distintivos para cada una. En esta medida, se puede decir que existe relación entre las cualidades superficiales de la madera, las emociones que provoca y la calidad percibida por los usuarios. La ponderación de las preferencias marcadas por los encuestados, determinan un orden que distingue a la madera de N. alpina como la más apreciada, seguida del $N$. obliqua, P. menziesii, Eucaliptus y Pinus radiata. Desde el punto de vista de la ingeniería kansei, se puede decir que el método aplicado es efectivo para conocer las preferencias emocionales de los individuos con capacidades visuales normales. Este método, además, puede ser replicado en investigaciones similares, por ejemplo, del ámbito de las cualidades superficiales de tableros revestidos o de la madera de otras especies, dejando abierta la posibilidad para realizar futuros estudios que permitan clasificar emocionalmente materiales existentes o en desarrollo.

\section{CONCLUSIONES}

La metodología empleada aborda la variable satisfacción emocional para estudiar la reacción basada en la percepción respecto de los cinco tipos de madera seleccionados. En la correlación entre emoción y cualidad superficial de la madera, se evidencia que las texturas visuales, color superficial, patrón decorativo, brillo, presencia de nudos, en sus variadas combinaciones, generan reacciones emocionales en los usuarios.

Resulta significativo que la ingeniería kansei, como metodología para determinar las emociones que diversos tipos de madera provocan en los proyectistas, permita determinar un orden jerárquico de preferencias basado en este criterio. Este orden permite conocer cuáles son las especies mejor valoradas por los entrevistados y establecer relaciones entre las cualidades superficiales de la madera, las emociones que provoca y la calidad percibida por los usuarios. Teniendo en cuenta estas valoraciones, los proyectistas podrán evaluar los usos de la madera al momento de decidir asuntos relativos a la satisfacción usuaria y plantearse la ideación interior de un hábitat acorde con ello.

Se considera parte relevante del procedimiento de este trabajo, la efectividad de una metodología para la obtención de resultados emocionales, centrado en la aplicación de diferencial semántico; integrado al proceso de vinculación de las cualidades superficiales de la madera y su presencia en espacios habitables. Esta metodología se complementa con la generación de pautas valiosas en términos de la constitución de una guía para los proyectistas. Se verifica su facultad para sistematizar la recogida de emociones, así como su eficacia al momento de la jerarquizarlas. De igual manera, la correcta interpretación de las mismas por parte de los proyectistas, entrega mayores proyecciones de aceptación usuaria vinculadas a satisfactores de uso. Esta definición conduce a la guía de parámetros susceptibles de ser incorporados en la fase de ideación de nuevas propuestas, con la salvedad de la necesidad de reiterar las 
mediciones cada vez que las especies cambian. La correlación entre emoción y cualidades superficiales de la madera según especies, debe mantenerse permanentemente actualizada.

Los hallazgos respaldan la proposición de que la ingeniería kansei permite recoger información para ofrecer soluciones más amables para los usuarios. Sin embargo, también puede contribuir en la implementación de investigaciones para conocer las reacciones emocionales de las personas frente a la madera de otras especies. Los resultados obtenidos dan valor a la importancia de una atención detallada a los componentes visuales relacionados, que se incorporan en la comunicación del espacio habitable en interacción con el usuario.

\section{RECONOCIMIENTOS}

Los autores agradecen a la Comisión Nacional Científica y Tecnológica, a través los proyectos FONDECYT $\mathrm{N}^{\circ}$ 11130394 y CONICYT Nº REDI170581.

\section{REFERENCIAS}

Bedolla, D. (2003). Diseño sensorial. Las nuevas pautas para la innovación, especialización y personalización del producto. Tesis doctoral, Universidad Politécnica de Cataluña. Recuperado de http://www.tdx.cat/handle/10803/6826

Centro Tecnológico para el Diseño y la Producción Industrial de Asturias [Prodintec]. (2011). Diseño Afectivo e Ingeniería Kansei, Guia Metodológica. Gijón: Fundación Prodintec.

Chávez, L., Hernández, C., \& Ruiz, C. (2010). Determinación de la calidad de la madera de construcción. Acta Universitaria, 20(2). doi: 10.15174/au.2010.71

Demir, E., Desmet, P., \& Hekkert, P. (2009). Appraisalpatterns of emotions in human-productinteraction. International Journal of Design, 3(2), 41-51.

Desmet, P. M. A., \& Hekkert, P. (2007). Framework of product experience. International Journal of Design 1(1), 57-66.

Desmet, P., \& Hekkert, P. (2009). Design\&Emotion. International Journal of Design 3(2, Special Issue Editorial), 1-6.

Desmet, P. M. A., \& Sääksjärvi, M. C. (2016). Form matters: Design creativity in positive psychological interventions. Psychology of Well-Being: Theory, Research and Practice 6, 7. doi: 10.1186/s13612-016-0043-5
Díaz-Vaz, J., Cuevas, H., Fernández, A., \& Inzunza, L. (2012). Características tecnológicas de Pseudotsuga menziesii creciendo en Valdivia. Bosque 9(2), 97-101

Fenko, A., Schifferstein, H., \& Hekkert, P. (2008). Which senses dominate at different stages of product experience? Proceedings of Design Research Society Conference 2008, Sheffield Hallam University, Sheffield, UK, 16-19 July 2008.

Fenko, A., Schifferstein, H., \& Hekkert, P. (2011). Noisy products: Does appearance matter?. International Journal of Design, 5(3), 77-87.

Fiore, A., \& Kimle, P. (1997). Understanding aesthetics for the merchandising and design professional. New York: Fairchild.

Fokkinga, S., \& Desmet, P. (2012). Meaningful mix or tricky conflict? A categorization of mixed emotional experiences and their usefulness for design. Proceedings of 8 th International Design and Emotion Conference, London 2012 Central Saint Martins College of Art \& Design, 11-14 September 2012.

Goleman, D. (2003). Emociones destructivas. Cómo comprenderlas y dominarlas. Barcelona: Vergara Grupo Zeta.

Hekkert, P., Snelders, D., \& van Wieringer, P. (2003). Mostadvanced, yet aceptable: Typicality and novelty as jointprediction of aestheticpreference in industrial design. British Journal of Psycology, 94(1), 111-124.

Huber, A., \& Trecaman, A. (2004). Eficiencia del uso del agua en plantaciones de Pinus radiata en Chile. Bosque, 25(3), 33-43. doi.org/10.4067/S0717-92002004000300004

Instituto Nacional de Normalización (1974). Madera-Terminología General. Santiago: Norma Chilena Oficial NCh173.Of73

Jordan, P. (2000). Designing pleasurable products. An introduction to the new human factors. Londres: Taylor and Francis.

Ludden, G., \& Schifferstein, H. (2007). Effects of visual auditory incongruity on product expression and surprise. International Journal of Design 1(3), 29-39.

Nagamachi, M. (1995). Kansei engineering: A new ergonomic consumer-oriented technology for product development. International Journal Industrial Ergonomics 15, 3-11. doi.org/10.1016/0169-8141(94)00052-5

Nagamachi, M. (Ed.). (2011). Kansei/ Affective Engineering. Boca de Ratón: CRC Press, Taylor \& Francis Group.

Özcan, E., \& van Egmond, R. (2009). The effect of visual context on the identification of ambiguous environmental sounds. Acta Psychologica, 131(2), 110-119. doi: 10.1016/j.actpsy.2009.03.007 
Osgood, C.E., Suci, G.J., \& Tannenbaum, P.H. (1957). The measurement of meaning. Urbana: University of Illinois Press. doi: 10.4236/ajcm.2013.33030

Rognoli, V., \& Levi, M. (2005). Materiali per il design: espressività e sensorialità, Milano: Polipress.

Rognoli, V. (2010). A broad survey on expressive-sensorial characterization of materials for design education. Middle East Technical University Journal of The Faculty of Architecture, 27(2), 287-300. doi: 10.4305/METU.JFA.2010.2.16

Sakuragawa, S., Miyazaki, Y., Kaneco, T., \& Makita, T. (2005). Influence of wood wall panels on physiological and psychological responses. Journal of Wood Science, 51(2), 136-140. doi.org/10.1007/s10086-004-0643-1

Schifferstein, H. N. J. (2006). The perceived importance of sensory modalities in product usage: a study of self-reports. Acta Psychologica, 121, 41-64. doi:10.1016/j.actpsy.2005.06.004

Schifferstein, H. N. J., \& Hekkert, P. (2011). Multisensory aesthetics in product design. In F. Bacci \& D. Melcher (Eds.), Art and the senses, 529-555. Oxford: Oxford University Press.

Schueller, S. M., \& Parks, A. C. (2014). The science of self-help: Translating positive psychology research into increased individual happiness. European Psychologist, 19(2), 145-155. doi.org/10.1027/1016-9040/a000181

Stein, B., \& Meredith, M. (1993). The Merging of the Senses. Cambridge: MITPress.

Tractinsky, A. S., Katz, D., \& Ikar, D. (2000). What is beautiful is usable. Interacting with Computers 13, 127-145. doi: org/10.1016/S0953-5438(00)00031-X

Valencia, A., Mugge, R., Schoormans, J. P. L., \& Schifferstein, H. N. J. (2014). Challenges in the design of smart productservice systems (PSSs): Experiences from practitioners. En E. Bohemia, A. Rieple, J. Liedtka, \& R. Cooper (Eds.) Proceedings of 19th Conference of DMI: Academic Design Management Conference. Boston: DMI.

Villanueva, J., Sampedro, A., de Ávila, I., \& González, D. (2013). Aplicación de técnicas de ingeniería afectiva, micromecanizado y fabricación aditiva al diseño de texturas capaces de transmitir sentimientos y emociones predefinidas. Gijón: Fundación Prodintec.

Viña, F. (1997). La madera como materia de expresión plástica. Análisis estructural $y$ tratamiento en escultura para interior $y$ exterior. Disertación Doctoral, Universidad de La Laguna, Tenerife. Recuperado de https:/ / dialnet.unirioja.es/servlet/tesis?codigo $=691$

Wong, W. (1991). Fundamentos del diseño bi y tridimensional ( $\left.7^{\mathrm{a}} \mathrm{ed}\right)$. Barcelona: Editorial Gustavo Gili.

Manuscrito recibido el 20 de abril de 2017

Aceptado el 17 de abril de 2018

Publicado el 24 de enero de 2019

Este documento se debe citar como:

Alarcón C., J., Llorens V., A., \& Ormeño B., G. (2019). Ingeniería kansei aplicada a un estudio referido a cinco maderas comerciales de Chile. Madera y Bosques, 25(1), e2511553. doi: 10.21829.myb.2019.2511553

Madera y Bosques por Instituto de Ecología, A.C. se distribuye bajo una Licencia Creative Commons Compartirlgual 4.0 Internacional. 